\title{
Experimental investigation and performance comparison of a single OWC, array and M-OWC.
}

\author{
S. Doyle, G. A. Aggidis
}

\begin{abstract}
Ocean wave energy continues to develop through innovation and a growing number of collaborations around the world. With the vast resource of wave energy on our doorstep it remains a focal point in ocean energy engineering with great potential. In order for wave energy to become more competitive and a serious player in the renewable energy mix, such innovations should not only benefit the wave energy sector but also other technological applications by providing attractive options for synergies on novel projects.
\end{abstract}

This paper concerns the experimental investigation of Oscillating Water Column (OWC) Wave Energy Converter (WEC) technology and its potential as a Multi-Oscillating Water Column (MOWC). This research investigation utilises a progressive and pragmatic experimental modelling approach, by cross comparing the models of a single standalone OWC, an OWC array and finally a modular M-OWC under the same environment and test conditions. Performance and characteristic responses are analysed while varying the values of OWC spacing, damping and wave conditions. The results indicate that the spacing of OWC chambers affects significantly the performance of an MOWC. While performance improves with increasing the spacing, the efficiency of the M-OWC is greater than that of a single OWC or OWC array at reduced spacing values. In addition, the results indicate that an OWC array is less efficient by having individual power take-off systems operating in isolation as opposed to the modular M-OWC.

Keywords: Wave Energy, Renewable Energy, Ocean Energy, Oscillating Water Columns, Wave Energy Converters, Experimental Modelling,

\section{Introduction}

\subsection{Background}

Ocean renewable energy research has never been more important than in today's fight against climate change with the political pressure around the globe inevitably pushing for development. Although wave energy is still in its infancy compared to other forms of renewable energy, and even other forms of ocean energy like tidal energy, there remain a largely untapped resource and potential that continually reinforces the need for wave energy to play a big role in the development of the renewable energy mix. The UK alone has a wave energy resource potential between 250 and $600 \mathrm{TWh} /$ year [1].

Several extensive reviews of various Wave Energy Converter (WEC) technologies can be found in literature [2]. Of the main WEC categories, the Oscillating Water Column (OWC) is one of the most popular due to its robustness, simplicity and versatility. The harvesting of ocean wave energy with an OWC is not a new idea; it dates back to 1965 in Japan where a fairway and weather buoy rated at $70 \mathrm{~W}$ and $120 \mathrm{~W}$ respectively, were powered by the waves [3]. Since then, a large number of WECs have been patented and developed at small scales but a very small number have reached full-scale trials never mind commercial deployment primarily due to the lack of competitiveness resulting in high capital costs and financial risk.

As defined in [4], aside from the OWC as a single standalone unit, Multi-Oscillating Water Columns (M-OWCs), which are essentially multiple OWCs coupled together, can be subcategorised into different systems: OWC array, segmented M-OWC and modular MOWC. It is widely known that ultimately, as with wind and tidal energy, WECs must be deployed on a large scale or in multiples as either arrays of individual devices or coupled - 
like segmented or modular M-OWCs for example - in order to benefit from the high density of the energy resource and the greater economic benefits of large scale.

The cost of energy production is quantified by an expression called the Levelised Cost of Energy (LCOE), which is dependent on the capital and operational costs, but more importantly with greater significance the annual energy production [5]. At larger scales the opportunity for integration with other applications increases, which also lowers the capital costs component of the LCOE.

The versatility of the OWC is still largely untapped with the majority of devices deployed having similar characteristics in terms of operating systems and even applications. Versatility and diversity is achieved in most existing M-OWCs concepts in general, with a wide range of applications and variations. The number of M-OWCs both conceptually and in research is lower in comparison to single OWCs, however, recent trends in research are proving that M-OWCs, or multiple OWC deployment at least, is gaining traction [4].

Most M-OWC concepts and devices are very large structures, which yields great opportunities for deployment diversification. Examples of these can be seen in existing concepts with WECs integrated into offshore wind turbine structures [6], breakwaters [7] and general offshore multi-use floating platforms [8]. Additional concepts don't only include OWCs but also point absorbers and overtopping devices integrated with breakwaters [9]. Such innovation and identification of these alternative applications and synergies are necessary to make wave energy more competitive and viable option for future investment.

\subsection{Modelling of OWCs and MOWCs}

Small scale single OWCs have been well modelled both experimentally and numerically. Investigations into shape optimisation and performance have been ongoing with the majority being fixed or shore based devices [10-13]. Fewer experiments have been carried out with floating OWCs, likely due to the added complexity in general. Especially with larger scaled or more dynamic models, this is also due to the provision required of the correct additional air volumes to satisfy compress compressibility scaling either with larger structures or the use of auxiliary air tanks $[14,15]$.

Albeit less often, the OWC array has been studied concerning optimal spacing, layout and/or geometrics mainly through analytical or numerical methods [16-19], but also experimentally [20], such as tests concerning array orientations, for which a terminating array proves to be superior [21].

Concerning the terminating array, multiple OWCs, not necessarily as an M-OWC, but in integrated applications such as in fixed shoreline defence or breakwater applications are becoming popular as a research topic as can be seen in the modelling by Refs. [22, 23]. The OWC array as an M-OWC in large floating breakwaters is also becoming relevant with recent work in Ref. [24, 25].

A variety of different M-OWCs, both open systems [26] and closed systems, have been modelled experimentally despite the complexity of the number of OWCs and structure sizes. Closed system modular MOWC device details and their modelling are reviewed in Ref. [4], and include the Seabreath [27], ShoreSwec [28], KNSWING [29], the Leacon [30] and more. These modular MOWCs have only been modelled as complete and rigid structures for purposes of concept verification and performance analysis. Hence, optimisation of certain significant parameters like those considered in this study (e.g. chamber spacing) cannot be easily studied.

Out of all experimental data obtained for every device tested, the comparison of these devices and the method of comparison can be ambiguous. The Capture Width Ratio (CWR) is a value commonly used to express capture efficiency of a WEC, which is the ratio of the power captured and the power in the width of the wave equal to the width of the device or capture width. An extensive review by Ref. 
[31], compares CWR values across a large number of devices for which such data has been published. While for some cases this could offer a fair comparison and be acceptable, cross-comparing models with respect to this value can add uncertainty especially considering the variations in methodology throughout literature.

Ultimately, a reliable method of cross model comparison would involve keeping as many aspects of the experimental modelling constant i.e. wave tank, environment, data acquisition and processing, scale of geometry etc. Here lays the premise of this research. This work, as a part of a larger research project, is intended to build fair comparative models starting from a single OWC, progressing onto an OWC array, then an M-OWC and beyond in order to investigate their operational characteristics at their optimal performance with the given conditions and geometries. Fair comparisons between models can be drawn from both the data and observation on aspects of response and performance, which all adds confidence in the conclusions of this pragmatic approach.

\subsection{Overview}

This paper is divided into four sections; Section 2 provides an overview of the experimental set-up with details of the facilities, instrumentation, geometries and methodology. In Section 3, the results are presented and discussed with comparisons in damping effects on the single OWC and MOWC (Section 3.1), the chamber spacing effects for the OWC array and M-OWC (Section 3.2) and overall performance of the three models in Section 3.3. Finally conclusions of the investigations are summarised Section 4.

\section{Experimental Set-up}

\subsection{Experimental Facilities}

All experiments were conducted at the Lancaster University Renewable Energy Group (LUREG) wave tank facility [32-34]. The tank is $12 \mathrm{~m} \mathrm{x} 2.5 \mathrm{~m}$ with an adjustable depth owing to a moveable floor. Adjustable beam structures span across the width of the tank to support the fixed models and wave probes. Waves are generated with seven paddles, which also provide absorption for any reflection. A mesh-type beach $2 \mathrm{~m}$ in length at the opposite end of the tank absorbs the transmitted wave. A set-up schematic can be seen in Figure 1.

\subsection{Wave Runs}

With a 1:50 scale model, wave conditions were prescribed according to the limitations of the tank, namely the frequency range of $0.7-$ $1.4 \mathrm{~Hz}$. With a maximum water depth of $h=$ $0.9 \mathrm{~m}$, most wave generated were of the deepwater regime with some intermediate waves according to the following condition[35]:

$$
\lambda / h \geq 0.5
$$

Additionally, steepness also dictated the wave height limits in order to avoid breaking crests at a steepness $s \approx 0.142$, where $s=H / \lambda$. Hence, a range of frequencies were used at various fixed wave heights as show in Table 1 . It should be reiterated here that this work is part of a larger project, hence, in these early stages and especially for concept verification, only regular waves are used and within the optimal frequency range of the WEC models.

Table 1 - Range of waves runs used at Lancaster University Renewable Energy Group Wave Tank.

\begin{tabular}{ccc}
\hline Wave Height, $H$ & Frequency, $f$ & $S_{\max }$ \\
\hline $0.06 \mathrm{~m}$ & $0.8-1.5 \mathrm{~Hz}$ & 0.086 \\
$0.08 \mathrm{~m}$ & $0.8-1.5 \mathrm{~Hz}$ & 0.115 \\
$0.1 \mathrm{~m}$ & $0.8-1.4 \mathrm{~Hz}$ & 0.1 \\
\hline
\end{tabular}




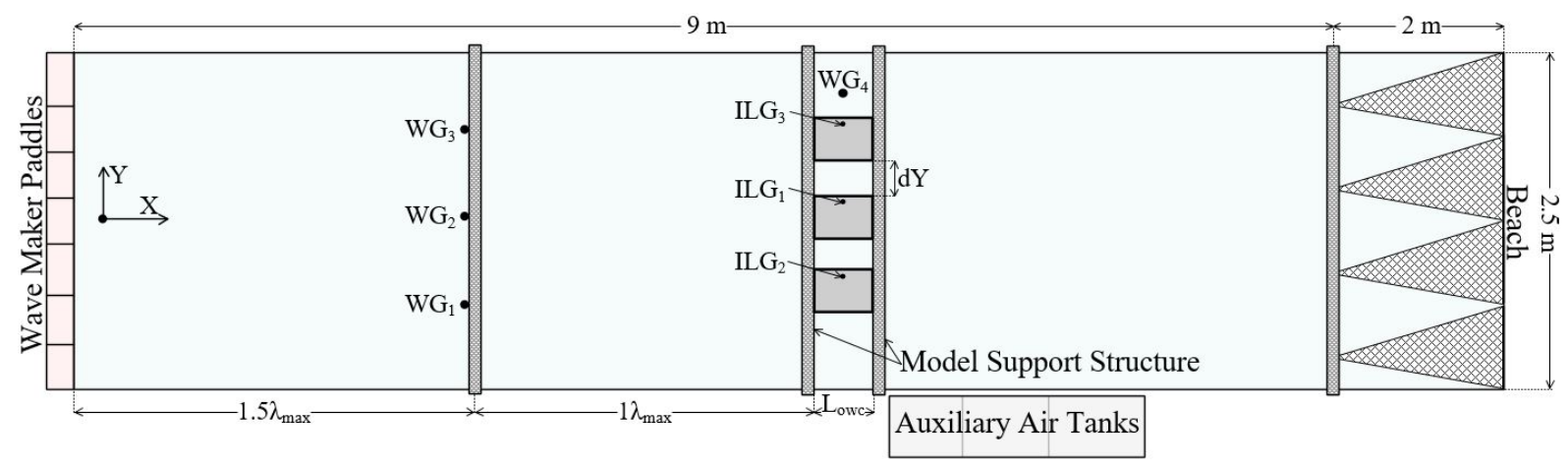

Figure 1 - Lancaster University Renewable Energy Group Wave Tank schematic detailing the important features including dimensions, model mounting area, wave gauge layout etc.

\subsection{Methodology}

Wave heights externally and internally to the chambers are measured with resistance water level gauges. For external wave amplitudes, three Wave Gauges $\left(\mathrm{WG}_{1-3}\right)$ are positioned $1.5 \lambda_{\max }$ from the device and $2 \lambda_{\max }$ from the wave paddles. A fourth gauge $\left(\mathrm{WG}_{4}\right)$ is positioned externally from the outer edge of the models and in a positioning that is aligned with the centre of the OWC/s. Each chamber includes an Internal Level Gauge ( $\mathrm{ILG}_{1-3}$ ), again, positioned on the centreline of the OWC/s with respect to the chamber length (Fig.1).

A total of three air pressure transducers are mounted in the lid of the OWC/s for individual chamber pressures (Fig. 2) and an additional one in the manifold for the M-OWC model (Fig.3). All data was acquired at a sample rate of $250 \mathrm{~Hz}$ and conditioned through a virtual instrument.

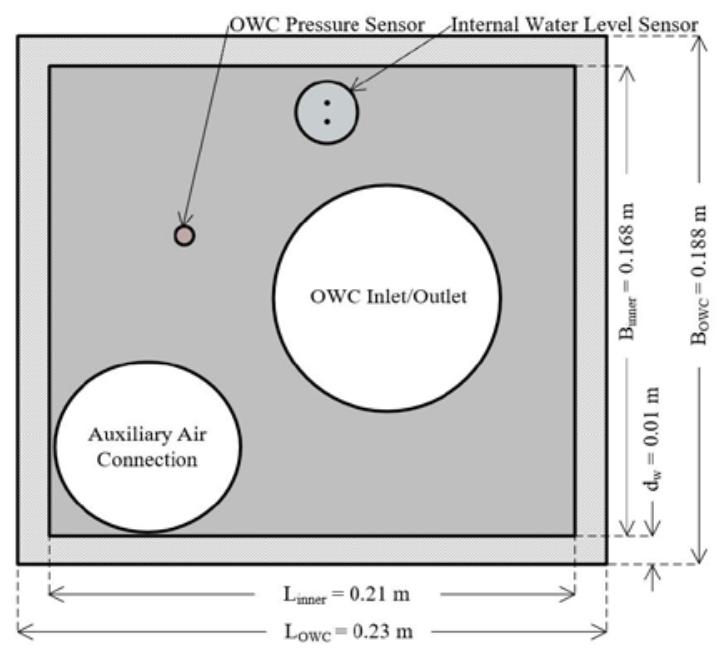

Figure 2 - Plan view of the OWC, its relevant dimensions, positioning of orifices and transducers.
For all experimental modelling, orifice plates were used to mimic the effects of PTO damping similar to that of an impulse turbine. These orifice plates are expressed as a percentage defined by their area, $A_{o}$, with respect to the water plane area of the OWC, $S$ :

$$
O=\frac{A_{o}}{S}=\frac{A_{o}}{\left(B_{\text {Inner }} L_{\text {Inner }}\right)}
$$

With the positioning of a pressure sensor upstream of an orifice plate, the differential pressure across the plate is calculated with respect to atmospheric pressure then used to find the flow rate through the orifice with the following empirical equation:

$$
Q_{o}=A_{o} V_{o}=A_{o} C_{d} \sqrt{\frac{2 \Delta p_{o}}{\rho_{\text {air }}(1-\beta)}}
$$

The discharge coefficient, $\mathrm{C}_{\mathrm{d}}$, is found with an empirical equation and is a function of the Reynolds number, Re, the geometrical ratio between the upstream diameter and the orifice diameter, $\beta$, and the distance to the of upstream, $\mathrm{L}_{1}$ and downstream pressure taps, $\mathrm{L}_{2}$ [36]:

$$
C_{d}\left(R e, \beta, L_{1}, L_{2}\right)
$$

Pneumatic power extracted from an OWC at the respective orifice plate is then found using the absolute value of the orifice flow rate:

$$
P_{o w c}=\Delta p_{o} \cdot\left|Q_{o}\right|
$$

Mean values of $P_{o w c}$ and $Q_{o}$ are found by integrating the time series of the variable over $N$ periods as shown in Equation 6 with the example of mean power, $P_{m}$ :

$$
P_{m}=\frac{1}{N T} \int_{t}^{t+N T} P_{o w c} d t
$$


The same methods is used to find $Q_{m}$ from $\left|Q_{o}\right|$.

Average peak power values, $\bar{P}_{\text {Peak }}$, are found by taking the average of the maximum values from $n$ half periods in order to account for the two power peaks in a single period from exhale and inhale processes:

$$
\bar{P}_{\text {Peak }}=\frac{1}{n} \sum_{i=0}^{n} P_{\text {max }}
$$

The same method is used to find the average max pressure, $\bar{p}_{\max }$, and average peak flow values, $\bar{Q}_{\text {Peak }}$, from peak values of $\left|Q_{\max }\right|$.

For the purpose of comparing the performance of the models tested, the Capture Width Ratio (CWR) is calculated in two forms; one with respect to the power in the wave across the effective width, $B_{E}$ and the second across the total width, $B$ T:

$$
\begin{aligned}
C W R_{E} & =\frac{P_{m}}{P_{\text {wave }} B_{E}} \\
C W R_{T} & =\frac{P_{m}}{P_{\text {wave }} B_{T}}
\end{aligned}
$$

The effective, or 'working' width, $B_{E}$ is essentially the product of number OWCs and the internal water plane width $B_{\text {Inner }}$, hence $B_{E}$ remains constant at $3 B_{\text {Inner. }}$ The total width, $B_{T}$ is the total width of the structure - the distance between opposite outer walls:

$$
B_{T}=3 B_{\text {owc }}+2 d Y
$$

For the average CWR, and average peak CWR values, the mean value, $P_{m}$ and average peak values, $\bar{P}_{\text {Peak, }}$, power values are used respectively.

\subsection{Tank Models}

All experimental models were fixed to support structures and constructed with $0.01 \mathrm{~m}$ thick Perspex sheets, which were fastened and sealed airtight. The models represent 1:50 scale of the full scale concept used as a geometrical baseline. Standard Froude scaling was used for geometric properties as can be seen in Table 2. Although the geometry of the OWCs could be scaled in this way, the scaling down of the systems air volume had to take into account the changes in compressibility between a large and small-scale volume. As found in literature, the compressibility-scaling factor $\varepsilon^{2}$ should be used instead of the Froude equivalent for volumetric scaling, $\varepsilon^{3}$ (Table 2) [37]. This meant an additional $0.216 \mathrm{~m}^{3}$ of air volume per OWC had to be provided without compromising the scaled OWC geometry, which was done so with the use of auxiliary tanks for each OWC partially filled with water and connected with flexible hoses directly from the lid of the OWC (Fig 2).

\subsubsection{Single OWC}

This single OWC was modelled under equal conditions in order to provide an accurate comparisons between models. Hence, the single OWC serves as a baseline with the same operational characteristics as opposed to using other external devices or external experimental data for comparison.

As with a typical mechanical system with a mass, damper and spring, this system has a resonant response. This can be found through experimental observation, as well as from empirical models based on geometry, such as in Equation 11 derived by Ref [38]:

$$
f_{n}=\left(2 \pi \sqrt{\frac{D+0.41 S^{0.5}}{g}}\right)^{-1}
$$

Such empirical equations allow an estimate of the resonant frequency based on geometrical features helping to ensure testing is possible in this facility with this geometrical scale. As these experiments are motivated by concept verification, the testing is carried out around this resonant and optimal frequency point. 
Table 2 - Dimensions of the OWC geometry for full scale and $50^{\text {th }}$ scale, and scale factors for each dimension.

\begin{tabular}{cccc}
\hline \multirow{2}{*}{ Dimension } & $\begin{array}{c}\text { Scale } \\
\text { Factor }\end{array}$ & $\begin{array}{c}\text { Full } \\
\text { Scale }\end{array}$ & $\begin{array}{c}1: 50 \\
\text { Scale }\end{array}$ \\
\hline$L_{\text {Inner }}$ & & $10.5 \mathrm{~m}$ & $0.21 \mathrm{~m}$ \\
$B_{\text {Inner }}$ & & $8.4 \mathrm{~m}$ & $0.168 \mathrm{~m}$ \\
$D_{\text {OWC }}$ & $\varepsilon$ & $8.4 \mathrm{~m}$ & $0.168 \mathrm{~m}$ \\
$F_{\text {Inner }}$ & & $6.3 \mathrm{~m}$ & $0.18 \mathrm{~m}$ \\
$\lambda$ & $\varepsilon$ & - & - \\
$V_{\text {Air }}$ & $\varepsilon^{2}$ & $555.7 \mathrm{~m}^{3}$ & $0.222 \mathrm{~m}^{3}$ \\
$T$ & $\varepsilon^{0.5}$ & - & - \\
\hline
\end{tabular}

\subsubsection{OWC Array}

An OWC Array is classed as an M-OWC device if the multiple individual OWCs are part of, or mounted in the same structure. Hence, the OWCs operate in isolation with individual PTOs and generators but are combined in a common structure.

The array here essentially a row of OWCs in terminating configuration, where there front walls are aligned parallel to the wave crest (Fig.3). The array is studied to understand the effect of spacing between the OWCs and how the response in both terms of individual performance and also as a M-OWC total unit. These experiments are in the interest of building context for a breakwater; hence, spacing is only in direction of the wave crest terminating configuration (Fig.3).

Spacing in between chambers, $a$, is expressed as a function of $B_{\text {Inner }}$ - the internal width of the OWC:

$$
a=\frac{d Y}{B_{\text {inner }}}
$$

where $d Y$ is the distance between the edges of the water plane area of neighbouring OWCs (Fig.1). Spacing is increased at intervals of $0.5 a$ for the range of $a=0$ to $a=3$. The mounting structure across the width of the tank supports the OWCs and provides easy adjustment with respect to spacing.

\subsubsection{M-OWC}

As defined in literature, a Modular M-OWC has multiple OWCs providing airflow that converges prior to the PTO system. This could include a final air chamber like an accumulator, high/low pressure ducting, or

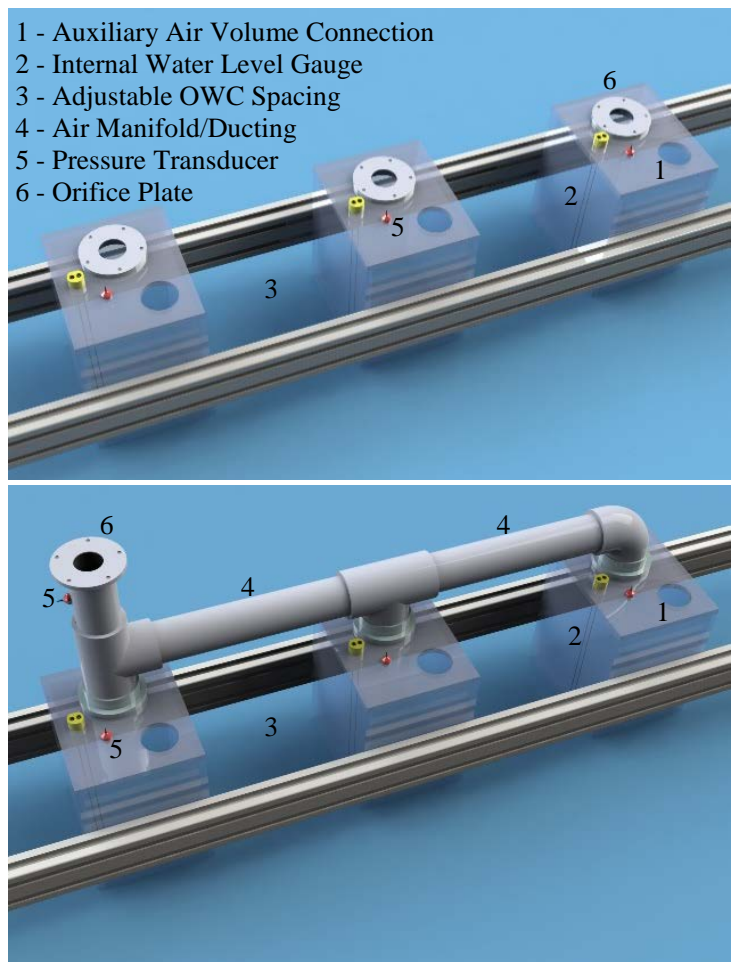

Figure 3 - Labelled renders of the design and layout of the OWC Array model (top) and M-OWC (bottom).

additional manipulation of the airflow e.g. a rectifying system. Most commonly, the Modular M-OWC concept is used in closed systems with unidirectional airflow and PTO systems [4]. In this case, bidirectional airflow is captured. Again, this sustains this pragmatic approach and makes the model comparative with the previous models with bidirectional airflow.

To achieve the coupling of OWCs, a duct or manifold is added, taking the flow from each chamber to a single pipe where an orifice plate is situated (Fig. 3). As with the OWC array, spacing could be easily adjusted, however, with the M-OWC, there was the added complexity of the replacing the ducting lengths at each interval and ensuring airtight joints.

This is by no means an optimal layout or design but is a result of the experimental model progression and keeping as many aspects constant, for example, OWC lid geometry. 


\section{Results and Discussion}

\subsection{Characteristics}

Throughout all experiments, it was found that the pneumatic power response was closer to the phase of the external wave than the response of the water level internal to the OWC chamber. An example of the time series of these values are plotted in Figure 4. Note the power, $P_{\text {owc }}$, response shows a peak twice per period as mentioned in Section 2.3 in the reasoning behind the form of Equation 7 .

As discussed in Section 2.4.1 the resonant frequency of an OWC can be estimated with Equation 11. Experiments were also ran with zero damping to determine the resonant frequency experimentally and observe the response of the geometry. This was achieved by removing the lids as if the OWC were an open moon pool. With the lid fitted and the inclusion of damping, the resonant point was found to be less prominent with in certain conditions with respect to the internal response. The internal water level heave response is characterised by the Response Amplitude Operator (RAO), which is the ratio of the amplitude of the response and the amplitude of the incident wave. The RAOs can be seen for zero damping (100\%) in Figure 5.

The estimate of the resonant frequency using Equation $11(\sim 1 \mathrm{~Hz})$ matches a peak found in most of the data presented, however, a more prominent peak is often found in the range of $0.92-0.94 \mathrm{~Hz}$. For the purposes of the experimental work in this paper, the frequency

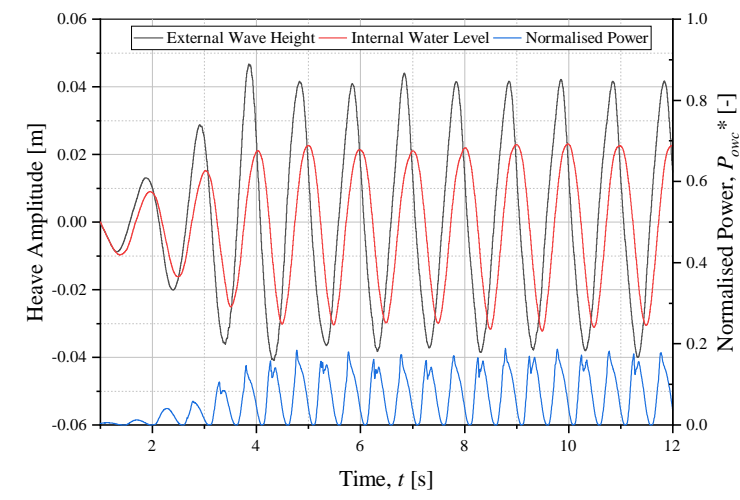

Figure 4-Example of a time series plot of internal water level, external wave height and power history obtained from the single OWC experiments. around these points were used for all subsequent testing. This allows a comparison between models at their optimal operating range and a higher resolution performance curve.

The curves often contain a trough in-between the aforementioned two peaks. At this point during the experiments, some internal sloshing was observed, which can occur at certain frequencies - usually higher frequencies. Since the internal level gauge is mounted in the centre of the chamber, the sloshing results in a lower mean water level as the front and rear inner walls experience the higher amplitudes.

Damping is applied to the same geometry with orifice plates as explained in Section 2.3 The response to the orifice range $1 \%-3 \%$ is plotted with the $100 \%$, which represents zero damping (Fig.5). While more suppressed, the shape of the dampened RAOs follows the case of zero damping. As frequency increases, the responses converge as the relative wavelength shortens to induce severe sloshing. In general, as expected, results show the damping has and inverse effect on the RAO magnitude.

A high RAO response is positive but not at the expense of power extraction, which ultimately is a function of the pressure drop created.

Figure 6 shows the effects of damping on the maximum pressure during exhale and the average net pressure, $p_{\text {Net }}$, which is the sum of the average maximum and minimum pressures, expressed as:

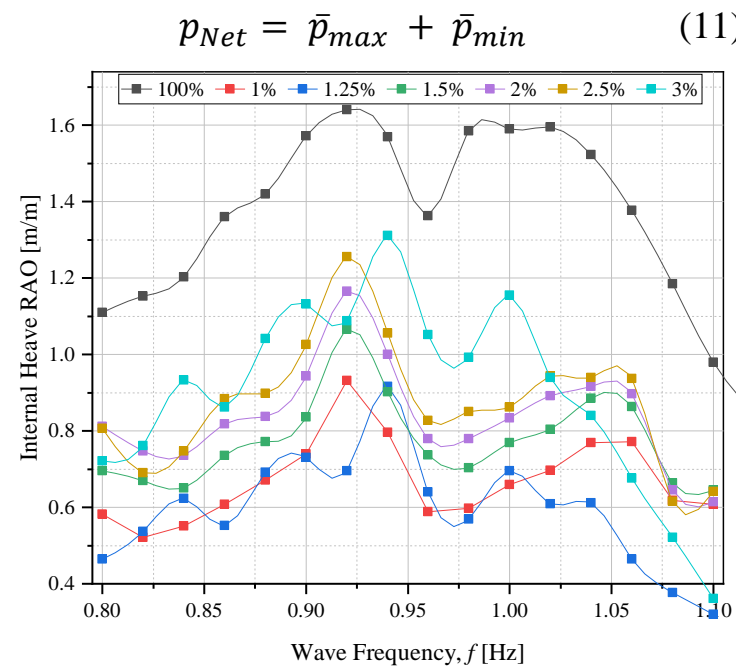

Figure 5 - Internal Heave RAO results from the single OWC orifice tests at $H=0.08 \mathrm{~m}$. 
With lower damping, peak pressures reduce and net pressures indicate that the inhale process in dominant. With orifice $1.25 \%$, peak pressures follow the trend with high values in between $1 \%$ and $1.5 \%$; however, the net pressures are closer to zero meaning a balance between inhale and exhale processes and therefore a higher mean power. Hence, similar magnitudes of power are generated in both processes per wave period - every half period - resulting in a higher mean power.

While constant damping is not suitable for 'real life' operation, without modelling a PTO system and in the absence of a control system, the perceived optimal damping orifice plate of $1.25 \%$ was chosen for all subsequent single OWC experiments.

In the case of the modular M-OWC, since there is effectively one orifice plate for three chambers larger orifice plates are required. Where $S$ is still the internal water plane area of a single OWC, it was estimated that 3.75\% would be appropriate (Eq. 2). A range of 3.5\% - $6 \%$ were tested to confirm these estimates (Fig. 7). Again, there is effectively a trade-off between the maximum pressure achievable and the RAO for optimal power - a behaviour also observed in the experiments. The estimated $3.75 \%$ was chosen to be used moving forward since it yielded high maximum pressures with a comparatively low cost to the RAOs.

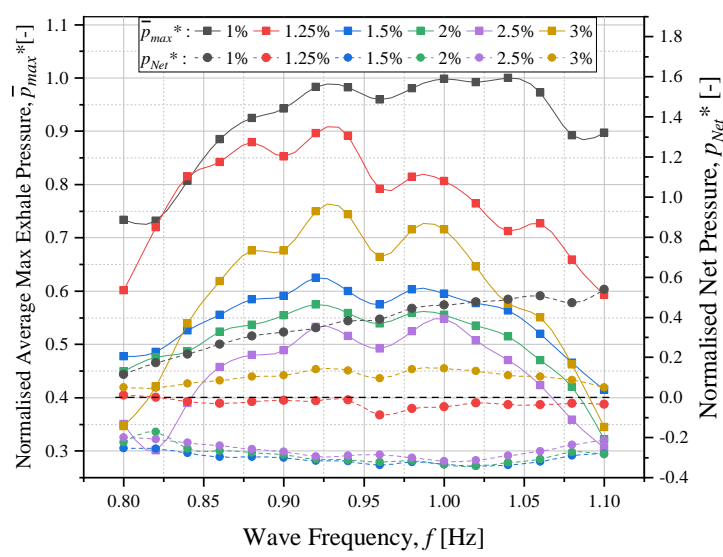

Figure 6 - Normalised values of average maximum exhale pressures and net pressures for different orifice plates from the single OWC

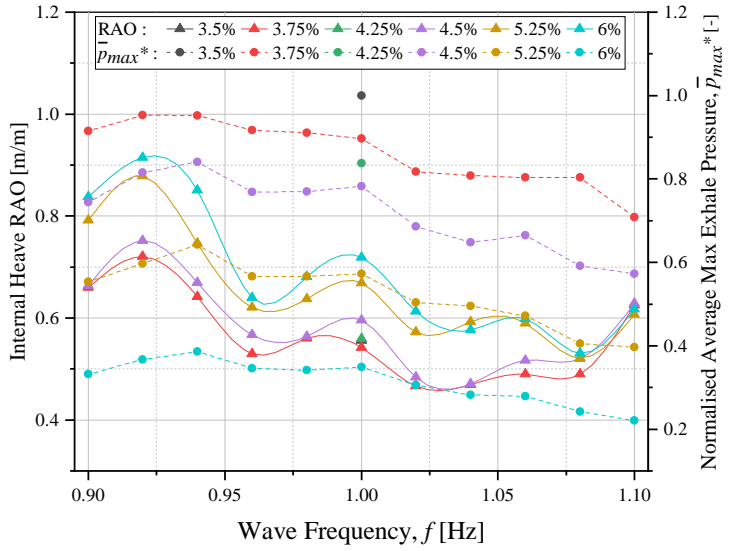

Figure 7 - Internal heave RAOs and normalised values of average maximum exhale pressure for different orifice plates from the M-OWC model.

\subsection{OWC Spacing}

With both the OWC Array and modular MOWC models and their chosen damping values, OWC spacings were adjusted from $a=$ 0 to $a=3$ in increments of 0.5 (Eq.10). Since is $\lambda_{\max }$ approaches the tank width $2.5 \mathrm{~m}$, affects where anticipated and closely monitored. No observations were made and there's confidence that the relatively small width of the tank did not affect these results. The wave angle was not adjusted and the responses of all OWCs were in phase suggesting there were minimal transverse effects. Greater uncertainties became apparent when spacing was increased to $\mathrm{a}=3.5$, resulting in the absence of this spacing in all subsequent tests hence, $a_{\max }=3$. The authors remain confident that despite the uncertainties of the effects of the tank width, the overall cross-model comparison conclusions of this study would not change.

Across all results and as is evident in Figure 8 where results are plotted for just the $1 \mathrm{~Hz}$ run, at $a<1$, performance is affected significantly. Improved performance from $a>1$ is negligible in most cases but can result in decreased performance as in the case of the M-OWC in Figure 8. Ultimately, it is ideal to have as many chambers as possible and hence the least spacing as possible. With a significant increase to $a=1$, and no certain improvement trends in performance from $a>1$, this is considered the ideal. 


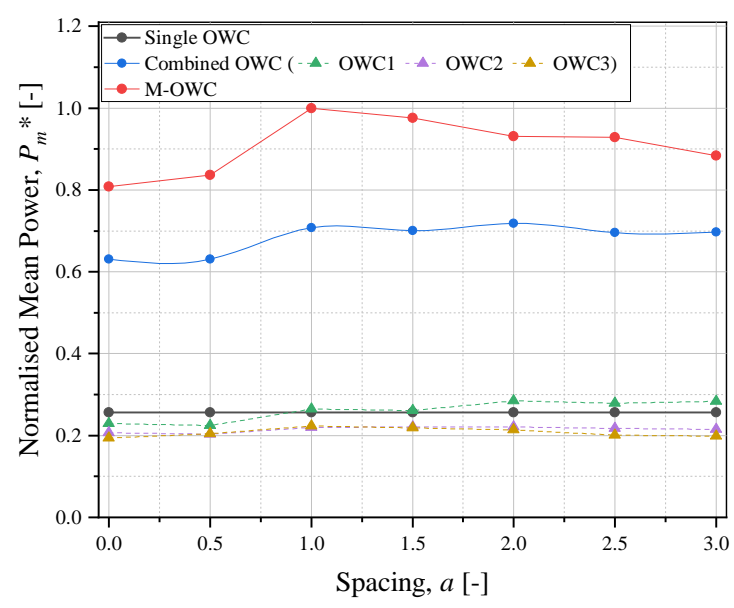

Figure 8 - Normalised values of mean power generated by the single OWC, the OWC array and M-OWC model with changing values of chamber spacing.

Against the single OWC, the individual OWCs of the OWC array perform differently. The centre OWC performs best at $a=1$ where the performance is almost equal to that of the single OWC. The outer OWCs, 2 and 3, do not improve with further spacing, only their impact on the centre OWC is evident. Hence, when $a<1$ these outer chambers are affecting the resource available for the centre chamber. Despite the distance between the OWCs increasing and therefore the greater the capture width, the outer chambers do not approach the performance of the single OWC.

Figure 8 also includes the combined power of the OWC array, which is simply the sum of the power generated by the individual OWCs. Since both outer OWCs under perform, the combined power is less or at very best the

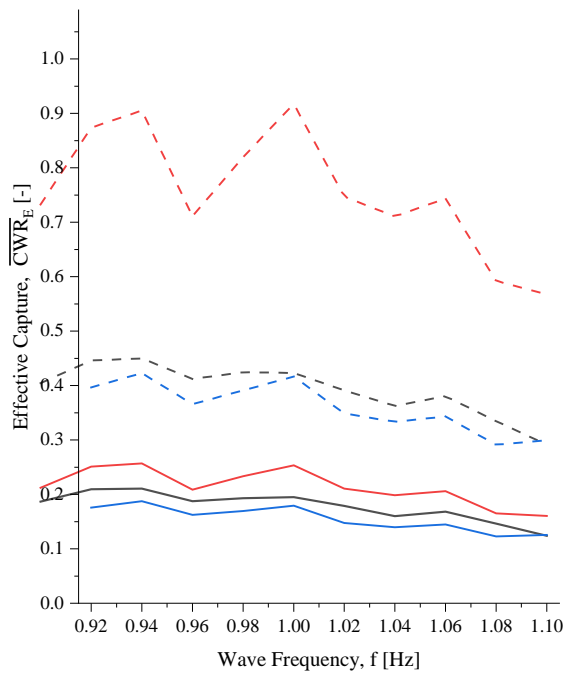

equivalent to three single OWCs in isolation. Since this behaviour is the same across other frequency points, it suggests that the effect of the resource absorbed by the centre chamber, could be to the detriment to the wave energy outside of this centre channel thus affecting the outer chambers.

Once again, the uncertainties of the effects of the tank width don't change this conclusion. Regardless of effects, the spacing improves at $a \geq 1$.

\subsection{Capture Performance}

The CWR is considered in two forms as detailed in Equations 8 and 9 and is plotted in Figure 9 in terms of average and average peak values against wave frequency for all models. For the OWC array, the power absorbed is the combined power of all three OWCs. Note that these models are not optimised and therefore these values are not to be compared with other devices but are only useful for the comparison between these models.

The OWC array does not exceed those values of the single OWC across the frequency range. The modular M-OWC is superior mainly with respect to average peak values. The effective capture width $\left(\mathrm{CWR} \mathrm{R}_{\mathrm{E}}\right)$ values are favourable toward both the OWC array and modular MOWC simply because the overall size of the

Figure 9 - Average and average peak values for the effective and total capture width values plotted as a function of wave frequency for the single OWC, the OWC array and M-OWC models.

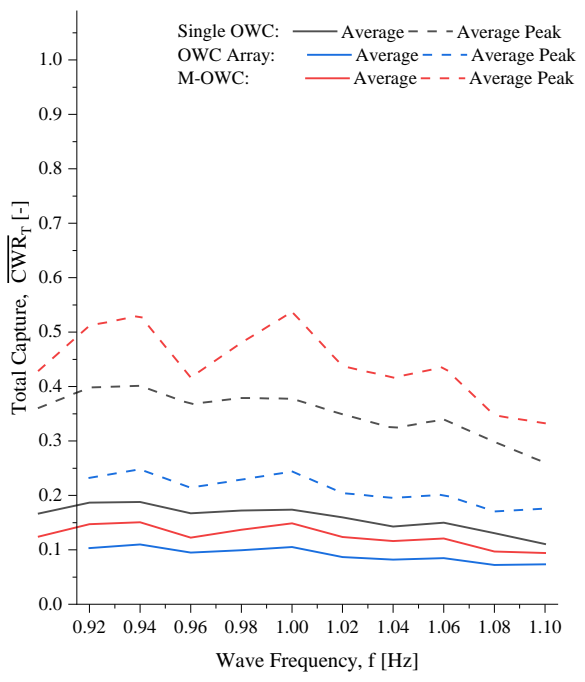



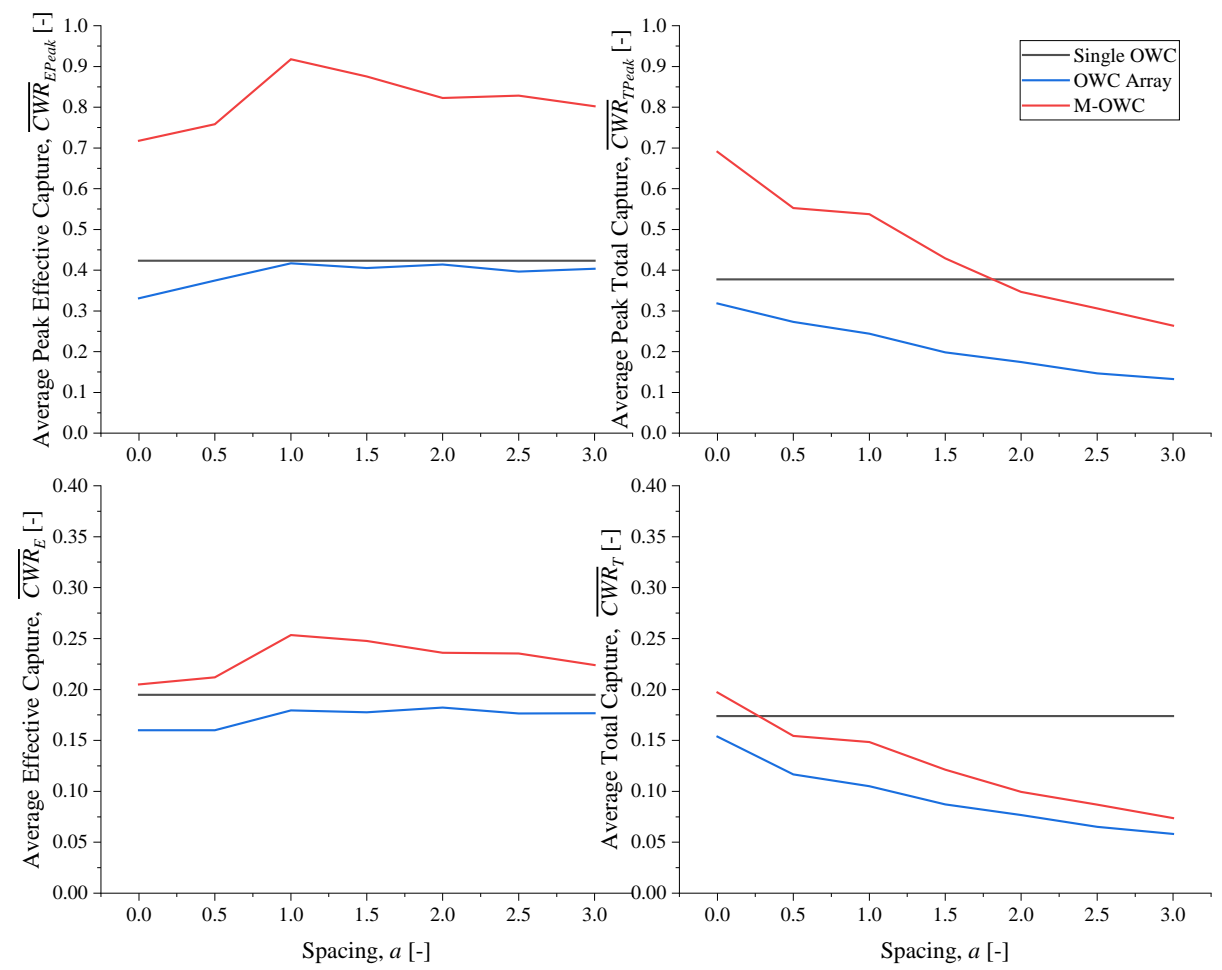

Figure 10 - Average and average peak values for the effective and total capture width values plotted against chamber spacing for the single OWC, the OWC array and M-OWC models.

structure isn't considered, only the combined widths of the OWCs (Eq.9). For the single OWC the difference between effective and total width is minimal since the difference in the width component between Equation 8 and 9 for $\mathrm{CWR}_{\mathrm{E}}$ and $\mathrm{CWR}_{\mathrm{T}}$ respectively, is only $0.02 \mathrm{~m}$ (twice the wall thickness). In the case of the M-OWC this proves that the device is more efficient with respect to the power in the width of the OWC. In truth, the resource absorbed by the OWCs is greater than the resource in the width of the OWC according to the observations made in the spacing analysis discussed above. However, the width of the resource and the sharing or distribution of the resource is difficult to analyse quantitatively.

In Figure 10, CWR values are plotted against OWC spacing for average and average peak values. Here the single OWC serves as a good baseline against the other models.

Again, the effective CWR is favourable toward both the OWC array and M-OWC by considering that the wave resource is only three chambers wide despite the spacing. Although the OWC array remains inferior to the single OWC, whereas the average peak effective CWR approaches 1 at the optimal spacing of $a=1$ for the M-OWC.

The M-OWC average peak values for the total CWR are far greater than the single OWC for when $a<1.5$. With respect to average total CWR, the single OWC is superior over the MOWC, but only when $a>0.5$. Hence, at the sub-optimal spacing, because of the decreased width of the structure, the M-OWC is more efficient that the single OWC.

Furthermore, despite spacing, the OWC array is always the least efficient especially with respect to the total CWR as spacing increases. As one would expect, with respect to the effective CWR, the OWC array follows the trend of the single OWC when $a>1$.

\subsection{A Final Word on Model Comparison}

The purpose of M-OWC WECs, in general, is to multiply the energy production of the device by a factor equal to the number of OWCs or greater if efficiency can be improved in the process. Moreover, with the airflow from all OWCs converging upstream of the PTO system, this should also mean that the flow rates should also multiply by a factor equal to the number of chambers - in this case three. 
In Figure 11, for the same input wave, the flow and power values are compared for the $\mathrm{M}$ OWC, OWC array and the single OWC. The values of the single OWC are multiplied by three to serve as useful benchmark.

It can be seen that the M-OWC exceeds in generating three times more flow and power than the equivalent three single OWCs with respect to both mean and average peak values.

The OWC array combined values manage to exceed the single OWC, but is inferior to the three single OWC equivalent across all variables. With respect to mean power, the array model generates at best 2.6 (not 3) times the single OWC. This suggests that poor performances are encountered with individual PTOs in the case of OWCs arrays.

Although these results do not consider the width of the resource (where $a=1$ ), the purposes and expectations of the M-OWC are delivered in the multiplication of the flow rates and power production by a factor equal to the number of OWCs and greater. This is something the OWC array fails to achieve, with the crucial difference being the PTO arrangement. These results therefore prove the concept of the M-OWC as being more efficient without considering the capture width in this case.

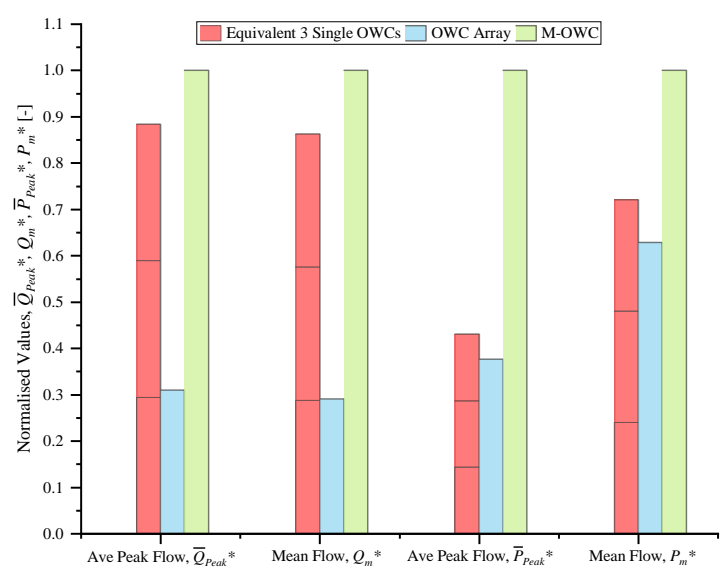

Figure 11 - Normalised values for average peak and average airflow, and average peak and mean power for the equivalent of three single OWCs, the OWC array and M-OWC models.

\section{Conclusions}

Three physical models have been investigated in experiments in which hydrodynamic and pneumatic performance has been analysed. Cross model performance comparisons have been carried out with respect to changing variables such as damping and chamber spacing.

The ultimate aim of this research was to investigate the potential benefits of a Modular M-OWC above an OWC Array, which is often considered in M-OWC concepts. This study was conducted with a pragmatic approach in order to provide a fair comparison between models as opposed to comparing the CWRs with abstract devices in different conditions, which can add great uncertainty.

With the ultimate goal to determine the performance differences and change in characteristics between a single OWC, OWC Array and M-OWC, the following conclusions can be drawn:

- For the single OWC with the detailed geometry, the orifice plate which creates the optimal damping for the best performance is the $1.25 \%$. This provides high RAOs with minimal cost to maximum pressures.

- Spacing of chambers for the OWC array and M-OWC has great effect on the power produced. Optimal is found to be when $a=$ 1 . When $a<1$ performance is reduced and when $a>1$, performance doesn't necessarily increase and can reduce but is ultimately less efficient.

- The individual OWCs in the OWC array at the very best match the performance of the single OWC despite changes in spacing. The OWCs improve after spacing of $a=1$, however, only the centre chamber approaches the performance level of a the single OWC. Taking into account the capture width, this means that the OWC array will always be less efficient, even at larger spacings that could not be catered for in these experiments, as the capture width is greater. 
- As the intentions of a modular M-OWC is to combine airflow from multiple chambers prior to the PTO to multiply flow rate and consequently power by a factor equal to the number of OWCs, the performance values should reflect this and have proven to do so. In fact, flow and power values are greater than three times the value recorded in the single OWC for a modular M-OWC with three chambers. This proves the concept viable and more so than an OWC array concept as an M-OWC, since the multiplication of OWCs has no benefit on performance due to the individual isolated PTO arrangement.

- For the same wave, the M-OWC produces higher flow rates and power than the OWC array and the equivalent of 3 individual single OWCs. However, with respect to effective and total capture width values, the greater the spacing the less efficient the WEC with respect to the total capture width. Despite this, due to the higher efficiency with respect to the effective width, at lower spacings, even though when $a<1$ the performance is suboptimal, the total capture width ratio exceeds the single OWC.

The aims and the objectives of this study were satisfied with the results found. Note that by no means are the models and their geometries currently optimised. The current geometry is a product of the progressive nature of the project and effort to keep as many aspects constant for fair comparative data.

Further work includes additional steps in the investigation of the superior performance of the M-OWC, further improvements and its potential in alternative or integrated applications.

\section{Acknowledgements}

This work is funded by Lancaster University through European Regional Development Fund, Centre of Global Eco-Innovation and industrial partners Waves2Watts Ltd.
All experimental work has been carried out at the Lancaster University Renewable Energy Group wave tank facility.

\section{References}

[1] Melikoglu, M. Current status and future of ocean energy sources: A global review. Ocean Engineering. 2018;148:563-73.

[2] Lopez.I, Andreu, J, Salvador, C, Martínez de Alegría, I, Kortabarria, I. Review of Wave Energy Technologies. Renewable and Sustainable Energy Reviews and The Necessary Power-equipment. 2013;27:413-34.

[3] Background Papers on Seafloor Engineering, Volume 2: Assembly of Engineering National Research Council; 1975.

[4] Doyle, S, Aggidis, G A. Development of multi-oscillating water columns as wave energy converters. Renewable and Sustainable Energy Reviews. 2019;107:(75-86).

[5] de Andreas, A, MacGillivray, A, Roberts, O, Guanche, R, Jeffrey, H. Beyond LCOE: A study of ocean energy technology development and deployment attractiveness. Sustainable Energy Technologies and Assessments. 2017;19:1-16.

[6] Perez-Collazo, C, Pemberton, R, Greaves, D, Iglesias, G. Monopile-mounted wave energy converter for a hybrid wind-wave system. Energy Conversion and Management. 2019;199.

[7] Howe, D, Nader, J, MacFarlane, G. Experimental investigation of multiple Oscillating Water Column Wave Energy Converters integrated in a floating breakwater: Energy extraction performance. Applied Ocean Research. 2020;97.

[8] Gubesch, E, Chin, C, Abdussamie, N, Penesis, I. The Hydrodynamic Performance of an Integrated Semisubmersible Platform with Wave Energy Converters: A Concept for Multi-Use Platforms. Australasian Coasts \& Ports. Hobart2019.

[9] Mustapa, M A, Yaakob, O B, Ahmed, Y M, Rheem, C, Koh, K K, Adnan, A A. 
Wave energy device and breakwater integration: A review. Renewable and Sustainable Energy Reviews. 2017;77:4358.

[10] Celik, A, Altunkaynak, A. Experimental investigations on the performance of a fixed-oscillating water column type wave energy converter. Energy. 2019;188.

[11] Ning, D, Wang, R, Gou, Y, Zhao, M, Teng, B. Numerical and experimental investigation of wave dynamics on a landfixed OWC device. Energy. 2016;115:326-37.

[12] Rezanejad, K, Guedes Soares, C, Lopez, I, Carballo, R. Experimental and numerical investigation of the hydrodynamic performance of an oscillating water column wave energy converter. Renewable Energy. 2017;106:1-16.

[13] Liu, Z, Xu, C, Qu, N, Cui, Y, Kim, K. Overall performance evaluation of a model-scale OWC wave energy converter. Renewable Energy.149:132538.

[14] Benreguig, P, Murphy, J, Sheng, W. Model Scale Testing of the Tupperwave Device with Comparison to a Conventional OWC. Proceedings of the ASME 2018 International Design Engineering Technical Conferences \& Computers and Information in Engineering Conference. Madrid, Spain2018.

[15] Elhanafi, A, MacFarlane, G, Fleming, A, Leong, Z. Experimental and numerical investigations on the intact and damage survivability of a floating-moored oscillating water column device. Applied Ocean Research. 2017;68:276-92.

[16] Nihous, G C. Wave power extraction by arbitrary arrays of non-diffracting oscillating water columns. Ocean Engineering. 2012;51:94-105.

[17] Nader, J, Zhu, S, Cooper, P, Stappenbelt, B. A finite-element study of the efficiency of arrays of oscillating water column wave energy converters. Ocean Engineering. 2012;43:72-81.

[18] Konispoloatis, D N, Mavrakos, S A. Hydrodynamic analysis of an array of interacting free-floating oscillating water column (OWC's) devices. Ocean Engineering. 2016;111:179-97.
[19] Falcao, A F O. Wave-power absorption by a periodic linear array of oscillating water columns. Ocean Engineering. 2002;29:1163-86.

[20] Thiruvenkatasamy, K, Neelamani, S. On the efficiency of wave energy caissons in array Applied Ocean Research. 1997;19:61-72.

[21] O'Boyle, L, Elsaber, B, Whittaker, T. Experimental Measurement ofWave Field Variations around Wave Energy Converter Arrays. Sustainablitiy. 2017;9.

[22] Wan, C, Yang, C, Fang, Q, You, Z, Geng, J, Wang, Y. Hydrodynamic Investigation of a Dual-Cylindrical OWC Wave Energy Converter Integrated into a Fixed Caisson Breakwater. Energies. 2020;13.

[23] Zheng, S, Antonini, A, Zhang, Y, Greaves, D, Miles, J, Iglesias, G. Wave power extraction from multiple oscillating water columns along a straight coast. Journal of Fluid Mechanics. 2019;878:445-80.

[24] He, F, Huang, Z, Law, A W. An experimental study of a floating breakwater with asymmetric pneumatic chambers for wave energy extraction. Applied Energy. 2013;106:222-31.

[25] Howe, D, Nader, J, MacFarlane, G. Integration of Wave Energy Converters within Floating Offshore Structures. Australasian Coasts \& Ports Conference. Hobart2019.

[26] Shalby, M, Walker, P, Dorrel, D G. Modelling of the multi-chamber oscillating water column in regular waves at model scale. 4th International Conference on Energy and Environment Research, ICEER 2017. Porto, Portgual2017.

[27] Martinelli, L, Ruol, P, Fassina, E, Giuliani, F, Delmonte, N. A Wave-2-Wire Experimental Investigation of the New "Seabreath" Wave Energy Converter: The Hydraulic Response. Coastal Engineering. 2014.

[28] Joubert, J R, Van Niekerk, J L. Recent developments in wave energy along the coast of southern Africa. 8th European Wave and Tidal Energy Conference. Upsala, Sweden2009.

[29] Nielsen.K. MARINET experiment KNSWING testing an I-Beam OWC attenuator. International Journal of Marine Energy. 2015;12:21-34. 
[30] Kofoed, J P, Frigaard, P B. Hydraulic evaluation of the Leancon wave energy converter 2008.

[31] Babarit, A. A database of capture width ratio of wave energy converters. Renewable Energy. 2015;80:610-28.

[32] Aggidis, G A, Taylor, C J. Overview of wave energy converter devices and the development of a new multi-axis laboratory prototype. IFAC PapersOnLine. 2017;50:15651-6.

[33] Bhinder, M A, Rahmati, M T, Mingham, C G, Aggidis, G A. Numerical hydrodynamic modelling of a pitching wave energy converter. European Journal of Computational Mechanics. 2015;24:129-43.

[34] Rahmati, M T, Aggidis, G A. Numerical and experimental analysis of the power output of a point absorber wave energy converter in irregular waves. Ocean Engineering. 2016;111:483-92.

[35] McCormick, M E. Ocean Wave Energy Conversion. New York2007.

[36] Institution, B S. Measurement of fluid flow by means of pressure differential devices - Guidelines for specification of nozzles and orifice plates beyond the scope of ISO 5167-1. 1998.

[37] Weber, J. Representation of non-linear aero-thermodynamic effects during small scale physical modelling of OWC WECs. 7th European Wave and Tidal Energy Conference. Porto, Portugal2007.

[38] Sheng, W, Alcorn, R, Lewis, T. Hydrodynamics of oscillating water column wave energy converters. 1st International Conference on Renewable Energies Offshore. Lisbon, Portugal2014. 


\section{Nomenclature}

\begin{tabular}{|c|c|c|c|}
\hline \multicolumn{2}{|c|}{ Roman letters } & \multicolumn{2}{|l|}{ Greek letters } \\
\hline$a$ & chamber spacing [-] & $\beta$ & up and downstream diameters ratio [-] \\
\hline$A_{o}$ & orifice area $\left[\mathrm{m}^{2}\right]$ & $\varepsilon$ & scale factor $[-]$ \\
\hline$B$ & width $[\mathrm{m}]$ & $\lambda$ & wavelength [m] \\
\hline$C_{d}$ & discharge coefficient [-] & $\rho_{\text {air }}$ & air density $\left[\mathrm{kg} \cdot \mathrm{m}^{-3}\right]$ \\
\hline$C_{t}$ & turbine flow coefficient [-] & & \\
\hline $\mathrm{CWR}_{E}$ & effective capture width ratio [-] & & \\
\hline $\mathrm{CWR}_{T}$ & total capture width ratio [-] & \multirow{2}{*}{\multicolumn{2}{|c|}{ Subscripts }} \\
\hline$D$ & OWC draft [m] & & \\
\hline$d_{w}$ & OWC Wall Thickness [m] & E & w.r.t. effective width \\
\hline$d Y$ & $\begin{array}{l}\text { spacing between inner OWC edges } \\
\text { [m] }\end{array}$ & Inner & OWC inner dimension \\
\hline$f$ & wave frequency $[\mathrm{Hz}]$ & $\max$ & maximum value \\
\hline$f_{n}$ & natural frequency $[\mathrm{Hz}]$ & $m$ & mean value \\
\hline$F$ & OWC freeboard [m] & $\min$ & minimum value \\
\hline$g$ & gravity $\left[\mathrm{m}_{\mathrm{s}} \mathrm{s}^{-2}\right]$ & Outer & OWC outer dimension \\
\hline$h$ & water depth [m] & $T$ & w.r.t. total width \\
\hline$H$ & wave height $[\mathrm{m}]$ & & \\
\hline$L_{\text {owc }}$ & outer length of OWC [m] & & \\
\hline$L_{1}$ & upstream pressure tap distance [m] & \multicolumn{2}{|l|}{ Superscripts } \\
\hline$L_{2}$ & downstream pressure tap distance $[\mathrm{m}]$ & $*$ & normalised value \\
\hline$n$ & number of half periods $[-]$ & & average value \\
\hline$N$ & number of periods [-] & & \\
\hline$O$ & orifice area ratio $[-]$ & & \\
\hline$P_{\text {owc }}$ & pneumatic power from OWC [W] & \multirow{2}{*}{\multicolumn{2}{|c|}{ Abbreviations }} \\
\hline$P_{\text {wave }}$ & power in unit width of wave $[\mathrm{W} / \mathrm{m}]$ & & \\
\hline$\Delta p_{o}$ & pressure drop across orifice $[\mathrm{Pa}]$ & CWR & Capture Width Ratio \\
\hline$p$ & internal air pressure $[\mathrm{Pa}]$ & $\mathrm{LCOE}$ & Levelised Cost of Energy \\
\hline$Q_{o}$ & orifice flow rate $\left[\mathrm{m}^{3} \cdot \mathrm{s}^{-1}\right]$ & LUREG & $\begin{array}{l}\text { Lancaster University Renewable } \\
\text { Energy Group }\end{array}$ \\
\hline $\operatorname{Re}$ & Reynolds number [-] & M-OWC & Multi-Oscillating Water Column \\
\hline$s$ & wave steepness [m/m] & OWC & Oscillating Water Column \\
\hline$S$ & OWC water plane area $\left[\mathrm{m}^{2}\right]$ & PTO & Power Take-Off \\
\hline$T$ & wave period [s] & RAO & Response Amplitude Operator \\
\hline $\mathrm{V}_{\text {air }}$ & air volume $\left[\mathrm{m}^{3}\right]$ & $\mathrm{ILG}_{\mathrm{i}}$ & Internal Level Gauge \\
\hline & & $\mathrm{WG}_{\mathrm{i}}$ & Water Level Gauge \\
\hline & & WEC & Wave Energy Converter \\
\hline
\end{tabular}

